\title{
The integration of intelligent transport systems in urban transport
}

\author{
T. Richter \& S. Ruhl \\ Department of Road Planning and Operation, \\ Technische Universitaet Berlin, Germany
}

\begin{abstract}
The global increase of mobility - especially in cities - and continuing climate change necessitates the decarbonisation of the transport system, the enhancement of the energy efficiency of transport and the implementation of innovative transport solutions. But the continuously growing volume of traffic can also result in congestion, air pollution and collapsed traffic systems, caused by fast growing populations, the lack of city planning and low financial budgets. An approach for managing traffic in urban areas is an intelligent transport system (ITS); especially large cities are forerunners for such solutions. At the same time, large events provide an opportunity to develop, implement and evaluate innovative transport solutions. The growing interest in international events like football championships, the Olympic Games or exhibitions require highperformance transport solutions and traffic concepts due to the large number of participants. In consequence of the international attention, for prestige purposes and not least the financial background, large events are drivers of sustainable developments in transport.

The focus of the current paper lies on new ITS solutions in the cities of Cape Town, Delhi and London. All three cities are part of the EU-funded project STADIUM (Smart Transport Applications Designed for large events with Impacts on Urban Mobility) which aims at improving the performance of transport services and systems made available for large events hosted by big cities. The project demonstrates ITS applications at three events: the FIFA Soccer World Cup (2010) in South Africa, the Commonwealth Games (2010) in India and the Summer Olympics (2012) in London.
\end{abstract}


The objective of the paper is to present the effects and impacts of the demonstration activities with regard to ITS applications for urban transport. Keywords: ITS, urban mobility, traffic management, large events, innovative transport, sustainability, energy efficiency.

\section{Introduction}

The European project STADIUM (Smart Transport Applications Designed for large events with Impacts on Urban Mobility) co-funded by the EC DG RTD (FP7) aims for improving the performance of public transport systems made available to a wide and differentiated range of users in the framework of large events hosted by big cities. The project take up of worldwide experience in transport during large events and the transfer of the know how to other cities worldwide with upcoming events. Innovative transport solutions in different fields of impact categories (e.g. transport efficiency, safety and security as well as environmental and urban aspects) are based on research activities. The ultimate goal of the project is the development of a handbook to guide future decision makers of large events through the field of transport management solutions based on ITS. The demonstration of the viability of European ITS technologies in emerging countries are also of main interest of the project. The project started in May 2009, involves 17 partners and lasts 4 years with a founding rate of about 3.5 Mio EURO.

The present paper focuses on the intermediate results of the three demonstrations in Cape Town (FIFA World Soccer Cup 2010), Delhi (Commonwealth Games 2010) and London (Summer Olympics 2012). Thereby the demonstrating subject respectively the kind of ITS solution can be differentiated in public transport management (Cape Town and Delhi) and general traffic management (London). Overall an overview about the status quo of transport systems, implemented systems and technologies and evaluation results in the three cities is given by this report.

\section{Transportation in Delhi, Cape Town and London}

The South African (SA) transport system is based on the taxi industry which supports unorganized and inefficient public transport. The Cape Town transport system is diversified in metered-taxis, bus lines, private minibus taxis (minivan) service and a bus rapid transit (BRT) which is under construction but already running between the airport and the city centre. Minibus taxis playing a major role in terms of number of passengers per annum. In time, transport through minibus taxis has established itself to fill the lack of public transport and has expanded more and more, thus becoming today the transport mode used by about $70 \%$ of passengers in South Africa. Because of the relevance of the minivan transport sector (para-transit) the SA demonstrator deals with an ITS application aiming to improve its performances through the development of a demand responsive transport service (DRT). 
The Delhi transportation system is predominately addicted to roads, whereas railways, including rapid transit systems like the Delhi Metro, serve only $1 \%$ of the total demand. About $60 \%$ of Delhi's total transport demand is covered by bus transport system according to the Delhi Development Authority [2]. Buses are the most popular means of transport. In the 20 years from 1981 to 2001, Delhi has seen a phenomenal increase in the growth of vehicles and traffic. The inevitable consequences are accidents, pollution, increased commuting time and waste of energy (Delhi Development Authority [3]). The today's traffic on roads is a heterogeneous mix of cycles, scooters, buses, cars and rickshaws competing with each other. The focus within this demonstration is on auto-rickshaws and taxis. Auto-rickshaws (popularly known as Auto) are an important and popular means of public transportation in Delhi, as they are cheaper than taxis. On the other hand the focus is on taxis though they are easily available but not an integral part of public transport.

The demonstration activities in both cities covering several domains such as traffic management, public transport monitoring and optimization, traveller services based on mobile communications for real-time traffic and public transport service information, mobile payment, special event information and booking, etc.

London is a very important transport hub in the United Kingdom. That affects both, the public transport and also the road traffic. The main part of the daily journey stages is covered by public transport (43\%), which is mainly divided into train, metro and bus systems. 34\% of the mobility needs in London are fulfilled by cars (TfL [4]). Nevertheless, the region of London has to manage the most motor vehicle flow in Great Britain with approximately 29,100 vehicles per day on all major roads and 5,400 vehicles per day on minor roads (DfT [5]). The high traffic density, the slight traffic speeds and the daily congestions in London are reasons for one of the world's largest and most ambitious plans to tackle urban congestions - the London Congestion Charge - which started in February 2003 (Blow et al. [6]). But this measure is just one step to prevent congestions. That's why London has a comprehensive requirement for a well-engineered traffic management system.

The demonstrator in London is geared to such a well structured traffic management system. The basis for operational procedures (e.g. rerouting of traffic) are actual information about the traffic situation. The implemented measures (autarkical detecting camera system) in London will cause a known traffic flow and reduce the impact of any unplanned incidents. Thereby the reaction time of the traffic operators will decrease and increase the quality of road traffic. The measures of the three demonstrators are described below.

\section{Applied systems and technologies}

The objective of the STADIUM project within the South Africa demonstration is the implementation of a DRT application as special service that will leave a legacy of improved public transport. The goal is to demonstrate that the DRT application as a tool which can improve passenger movements in cities and 
especially during large events and beyond. The research objectives of the South Africa demonstration is the analysis of the added value of the DRT service for the operator, service provider (or affected stakeholder) and customers, compared to the existing service. The demonstration aims for answering the question of transport performance, socio-economic impacts as well as improved impacts in terms of environmental impacts. The analysis of the user acceptance plays an important role when evaluating the implemented service.

The DRT software is an internationally tested and proven scheduling application. The software was adapted to specifically meet the operator-, service provider- and local South African and Cape Town conditions. The call centre with scheduling software and a communication interface is used to schedule the DRT vehicles and to communicate the required schedules to the vehicles. The implementation of a DRT service requires a set of soft- and hardware. The software application consists out of the DRT website, the DRT kiosk software and other future DRT booking mechanisms (e.g. SMS booking service). The implemented on-board equipment includes a GPS tracking solution which gives the operator and service provider live vehicle location information. A fully customisable fleet management application forms part of the DRT application. Fleet management reporting includes reports on operator service quality, vehicle usage, driver behaviour, etc. which gains insight in and ultimately improve the quality of the service. Additional insight and control over the operations is gained by monitoring unsolicited stops and/or passengers by means of the door sensor, GPS and camera equipment combination. Exception reports are generated which helps to electronically monitor the service level of the operator. For demonstration there are 20 vehicles which are used for last mile services.

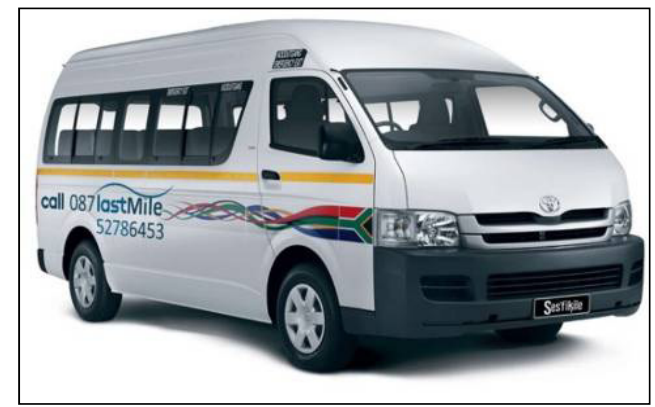

Figure 1: $\quad$ Last mile services.

The demonstrating vehicles (fig. 1) are with onboard equipment which:

- communicates the vehicle's GPS location to a call centre operator;

- provides a driver interface through which the driver can communicate with a call centre operator; 
- displays a schedule that the driver should follow including scheduled stops and passenger requests; and

- displays advertisements and marketing material to passengers on an LCD display inside the vehicle.

The objectives of the Delhi demonstration are the integration of Intelligent Transport System applications (ITS) for public transport which allows improving travel information and the quality of public transport. With the long term goal of an improved public transport service more passengers could be attracted which could reduce congestions and contribute to a sustainable transport. The research objectives of the demonstration are proving the concept of a planning tool on the basis of real-time monitoring of public transport and real-time passenger information, based on a multimodal database. The demonstration involved a number of public transport lines, such as metro lines, bus lines, para-transit services as well as control centres. For the demo purposes, a limited area has been considered in Delhi, addressing some Metro and BRT lines with about 200 GPS equipped buses and a sample of 30 para-transit vehicles and about 30 taxis. With regard to para-transit, taxis and auto-rickshaw (fig. 2) are considered as feeder services to these main transport systems.

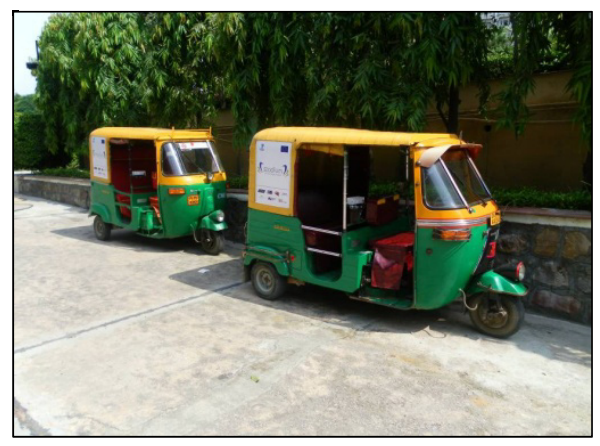

Figure 2: $\quad$ Stadium auto-rickshaw.

The demonstration in Delhi integrates four elements to provide its solutions:

- A control centre computer system to plan the public transport services and display real-time positions of vehicles and status of service. It also gives out passenger information in real time; integrates existing bus fleet based on GPS data with ability to collect about 200 GPS positioning data on BRT lines;

- demo bus equipped with an onboard equipment consisting out of GPS module, GPRS transmitter for real-time communication with the control centre, a WiFi connection to the depot facilities, automatic audio and visual announcements, video surveillance-and-recording (involving 4 colour-TV cameras), automatic passenger-counting systems, and 
message exchange between driver and control centre via a touch-screen driver terminal;

- taxi and para-transit vehicles equipped with on-board low-cost mobile devices;

- mobile phones for displaying public transport service information in real time, integrating data from the metro- and bus services.

A focus of the London demonstrator was to prevent congestions during the Summer Olympics 2012. London was faced with the big challenge of ensuring that the transport systems can cope with the enormous surge in demand, arising from the large number of event-related users (athletes, spectators, equipment, press etc.), while still catering for the needs of the everyday users. There 1450 traffic cameras across London deliver coverage in real time to the London Streets Traffic Control Centre (LSTCC). Operators observe the video images 24/7 while there is a limitation of how many staff can monitor cameras. The STADIUM demonstration has allowed for automatic notifications of congestion along the Olympic route network and around Olympic venues. The image recognition server was used to processes data from 12 existing traffic cameras (TfL [7]). At the STADIUM video server analysis tools are working with the incoming video streams and send an alert to the operators, if congestions are detected. In addition a new system of 6 smart cameras basically provided another set of intelligent eyes. The smart cameras detect congestions autarkical when vehicles in the monitored section are not moving for a time and send an alert and an image to the operators who are able to react to this situation with a standard set of measures (fig. 3). The output of both systems is the same (an alert to the operator) and the effects have to be evaluated in STADIUM project.

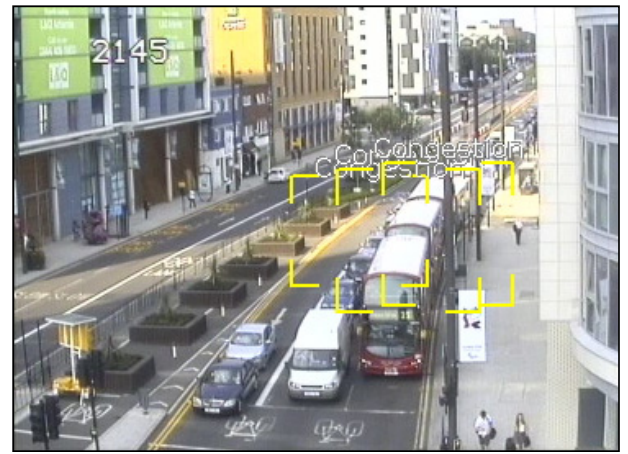

Figure 3: Detection of Congestion.

In the surrounding road network of the overall 18 cameras data are collected by inductive loops and licence plate recognition cameras. The originated relevant evaluation data are traffic flow and occupancy for the nearest inductive loops and journey times for specific links. In addition to this, an interview questionnaire 
will give information about the acceptance of the operators, who work with the assistance camera system.

\section{Evaluation approach}

The demonstrators address the task of improving the performance of transport systems in large cities during large events. Their common goal is to make the public transport system available to a wide and differentiated range of user's through the development of a set of services and tools to improve the information of the user and quality of the system. In addition to this, the London demonstrator gives an overview about the effects of intelligent transport management of the road traffic.

In the demonstrations different approaches and technologies were implemented which provides alternative transport management approaches through ITS. The objective of the research approach is to formulate a guideline for evaluation focusing the goal of the single demonstration, the system and technology applied and the method for collecting and analysing the data in terms of the different impact categories. Finally the made results will be evaluated against the defined objectives. Considering the valuable results of previous project documentations (e.g. MAESTRO, VIKING) and the new influences of the STADIUM challenges (large events) the evaluation approach was developed. Impact evaluation is an assessment or estimate of the impacts or effects of a measure, for example concerning safety, environmental conditions or transport efficiency, on the particular target groups (passengers, drivers, system operators, society, etc.) that are affected. The context in which the decision to implement a measure has been made should have a strong bearing on the impacts which need to be evaluated, the objectives against which they are measured and the target groups to be considered. Impact evaluations can be based on the results of direct observations, surveys, or calculations, e.g. modelling or simulation. An impact evaluation may provide input, for example, for related socio-economic policies assessment or may support the decision process directly (PLUTO [8]).

In the STADIUM project ITS solutions are used to improve urban transport. These solutions which are adjusted onto the site should realise the objectives set before. The objectives are part of the following impact categories:

- Transport Performance

- Social-Economic Impacts

Each impact category has a variety of potential objectives (measured by indicators). An appropriate choice of objectives was made due to the demonstration characteristics.

The overall research approach of the different demonstrations is mainly based on the MAESTRO-Guidelines for evaluation [9]. In addition to the traditional technical evaluation based on a static assessment of two states of things (the exante and the ex-post), the actual procedure for evaluation includes an assessment of the dynamic process of planning and implementation, in order to let emerge 
those factors which may affect the outcome of a policy related to the political, cultural, institutional, legal and financial background. Therefore four evaluation stages are implemented:

- the initial evaluation;

- the ex-ante evaluation;

- the process evaluation;

- the ex-post evaluation.

Taking into account the different impact categories mentioned above, related and measureable indicators were identified. With regard to measure and assess the impacts an experimental design was developed to realize reliable and comparable data within the different phases of evaluation.

\section{Results of the evaluation}

The results of the demonstration in South Africa enable to assess the STADIUM demonstration service before and during the 2010 FIFA world Cup in Cape Town. The assessment was made in terms of drivers and operators, passengers and also support systems and infrastructure, service offering and marketing.

The following information summarises inputs received about the drivers and operators from structured discussions and interviews with selected operators and drivers. They overwhelmingly agreed that is difficult or very difficult to get passengers in the off-peak period. While the minibus taxis are currently not scheduled, the drivers prefer scheduled services to non-scheduled ones. Drivers also prefer specific stops to load or offload passengers as opposed to the current arrangement where minibus taxi can stop at any point along the route. The following is a summary of inputs received with regard to the demand responsive transport system arrangements:

- Generally, they seemed indifferent with regard to the capabilities of the system to improve the way they plan their day.

- They are pleased about the capability of the system to help direct them to potential passenger locations.

- They were generally indifferent about the capability of the system to improve their income during peak and off-peak periods.

- Some thought the system has the capability to reduce their operating costs, while others were indifferent.

- They generally agreed that the system could potentially improve their overall working conditions. In order to improve working conditions even further, they thought the following areas of improvement were critical: (i) installation of operational navigator, (ii) provide mobile phone airtime to drivers to be able to interact with the customer directly, (iii) have the minimum pick up time to about 15 minutes in order to account for potential road traffic congestion, and (iv) give incentives to drivers who perform well. 
- They generally thought the system would improve services for customers. In their opinion, in order for the system to improve conditions for passengers even further, they identified the following as critical: (i) marketing of the service, and (ii) providing drivers with comprehensive customer service training.

The majority of the passengers interviewed during the FIFA SWC were foreign visitors to the City of Cape Town. Passengers were asked to evaluate the "Last Mile" service (a term used for marketing purpose) from a list of predetermined service attributes. The summarized results are the following:

- Service affordability was rated the most important attribute and passengers were generally satisfied with the fares.

- Of all the attributes, toll free phone call service, while important, it was rated as the least important relative to other service attributes.

- Passengers were particularly satisfied with punctuality due to the availability of vehicles on demand.

- Relative to other attributes, privacy was rated as the least performing.

However, passengers were satisfied with the service. Generally, a demand for the service from both operators and passengers does exist. Operators would like to use the system to improve their financial performance in the off-peak period and passenger would appreciate its convenience.

The demonstrator in Delhi aimed at showing the benefits of multimodal, integrated information to public transport users making their plan and trip more efficiently. In addition the defined goal the generation of timetables and real time forecast of bus arrival time at stops based on real time GPS localization of buses through the implementation of innovative ITS applications. The timeframe of field test was between the 1/9/2010 till 3/2011. There were 200 busses, 30 taxis and 30 auto-rickshaws equipped with the respective on-board-equipment. The overall number of operational vehicles is: 5000 busses, 5000 auto-rickshaws and 2000 taxis.

The number of interviews with passengers held was 150 and the number of interviews held with drivers was about 10 plus 10 operators. More than 35\% interviewees using public transport every day and more than $80 \%$ using public transport at least 3-4 times a week. A combination of different public transport modes would be used by more than $60 \%$ of the respondents.

In a before and after questionnaire the respondents answers regarding the service importance and the rating of the service quality are requested. For example the importance of the Service punctuality and frequency was rated high whereas the payment method and the service affordability were rated as less important. The results of the after questionnaire were in all cases better than in the before-questionnaire. Generally, the results of the passenger questionnaire show a positive attitude towards the implemented system. 
At the London demonstrator the data measurement spans over four scenarios. The timeframe was the following:

- two weeks before the Olympics (scenario without the autarkical congestion detection system),

- during the Olympics (scenario with the new system and with large events traffic demand),

- two weeks after the Olympics (scenario with the new system and without large events traffic demand),

- $\quad$ and an extended period during the Paralympics.

By comparing the scenario without the new camera system before the Olympics with the scenario with the new camera system after the Olympics it was possible to evaluate the general effects of that autarkical camera system. After that the general effects can be compared with the situation during the Olympics to get information about the effects during large events. The extended period was just an additional possibility to evaluate the system effects during large events with high traffic density and therefore special requirements to the traffic system.

The first analysis of traffic situation in the surrounding roads of the new camera system shows a comparable traffic flow in the considered scenarios. During large events there is the possibility of congestions in the night hours after finished events so that the period of low traffic intensity does not exist like in the ordinary course of the day. It can be noted that the type of incidents on the road network can vary significantly, because London is a highly populated city with large amounts of traffic. Especially during large events additional congestions can occur in times when events start or end. The autarkical detection of incidents is a possibility to support the work of traffic operator during large events and also in their daily work. Basis for a reliable detection of congestion and an efficient work of the traffic control centres are well designed parameters, which present a limit of acceptable traffic situations.

Additional results of the questionnaires, also regarding the operator point of view of the system implemented, will be analysed within the next period. The questionnaires will give an information of how the operator can react, how the incident was resolved and how quickly. This audit trail of operators work will complete the evaluation of traffic effects.

As a general effect it can be assumed, that the new system has a positive effect to the reaction times of the operators and thereby also to the traffic flow and journey times in London. A potential expanding of the described detection system may intensify the positive effects.

\section{Conclusion}

The analysis in Delhi and Cape Town has shown a clear picture of the passengers' expectation regarding public transport. The service affordability, frequency and the punctuality were rated high. These are correlating indicators to 
a high expectancy of travel time reliability. All of the mentioned indicators have been rated high after experiencing these services. Both, the Delhi as well as the Cape Town demonstration realised a high quality of service. There was no negative appraisal made by the customers. Even the drivers, although generally indifferent, draw the conclusion that the system and services provided could contribute to their daily working conditions and provide additional income. The implemented systems provide additional features for future service enhancements. Additional services (i.e. dial a ride for disabled, school groups etc) have the potential to access new markets through focussing different target groups. The analyses of the implemented systems have shown a high potential of managing transport in emerging cities via ITS. Although that, additional marketing effort and quality improvements of the service are crucial for raising the customers interests and acceptance. Finally, as a result a higher market penetration would have an increasingly positive impact on public transport performance with effects on the overall road transport.

The analysis in London was focussed at general traffic management, but can also affect an improved situation of street based public transport systems. The most benefit of an autarkical camera system is the possibility, that the traffic managing operators are able to focus their attention on other bottle necks in the road network, regardless of whether a large event occurs or not. Through a tested definition of traffic conditions a reliable autarkical detection of congestions is possible, which benefit in a quick realisation of countering measures.

Intelligent transport systems in general have high potential to increase the effectiveness of public transport systems and also the general traffic. This can affect a higher transportation quality in every traffic section. The implemented systems show a high user acceptance and are able to reduce journey times and traffic emissions. The implemented systems of the three STADIUM demonstrators are proved successful under special traffic conditions like large events. The results can be used for decision makers of upcoming large events and also in other large cities without large events. This is the first step to implement intelligent transport systems also in other large cities with high traffic density. The project demonstrated different opportunities for innovative and sustainable strategies and measures which will help to create efficient future traffic planning in cities with and without future events.

\section{References}

[1] Gupta, J.D., Nonmotorized Urban Transportation for Emerging Cities in India, Transportation Research Record, No. 1372, p. 85-89, Washington 1992

[2] Delhi Development Authority, Delhi Master Plan 2021, p. 97, New Delhi 2005

[3] Delhi Development Authority, Delhi Master Plan 2021, p. 89, New Delhi 2005

[4] Transport for London, Travel in London, Report 5, p. 25, London 2011 
[5] Department for Transport, Motor vehicle flow by road class and region and country in Great Britain annual from 2006, Road traffic statistics, Online. http://assets.dft.gov.uk/statistics/tables/tra0302.xls

[6] Blow, L., Leicester, A., Smith, Z., London’s Congestion Charge, Institute for fiscal studies, Briefing Note No. 31, 2003, Online. http://eprints.ucl.ac.uk /14932/1/14932.pdf

[7] Transport for London, Evaluation London Demonstration, Draft for D6.4 of STADIUM project, London, 2012

[8] PLUTO - Evaluation Planning Intelligent Transport Systems Handbook, Vaegverket, Borlaenge 2002, Online.

[9] http://www.durbit.se/Archives/ExternalPDF/MIP2004/Domain8/Miscellane ous/PLUTO_eng.pdf

[10] MAESTRO Guidelines - Monitoring Assessment and Evaluation of Transport, Brussels, 2000, Online. http://www.transport-research.info /Upload/Documents/200310/maestro.pdf 